\title{
Magnetic Properties of Hexagonal Graphene Nanomeshes
}

\author{
M. ZWIERZYCKI ${ }^{a, *}$ AND D. RYNDYK ${ }^{b}$ \\ ${ }^{a}$ Institute of Molecular Physics, Polish Academy of Sciences, M. Smoluchowskiego 17, 60-179 Poznań, Poland \\ ${ }^{b}$ Bremen Center for Computational Materials Science, University of Bremen,
}

Am Fallturm 1, 28359 Bremen, Germany

\begin{abstract}
Graphene nanomeshes are the nanostructures consisting of graphene flake with a regular pattern of antidots (holes) punched through it. Thanks to the energy gaps opening in electronic spectrum, nanomesh-based transistors offer improved $I_{o n} / I_{o f f}$ ratio of the collector current while supporting up to 100 larger driving currents than nanoribbon-based devices. In this paper the electronic and magnetic structure of graphene nanomeshes with hexagonally shaped antidots was studied. It has been found that the internal zigzag edges support magnetic moments and that lowest energy magnetic configuration is antiferromagnetic. The density of states calculated for ground state configuration exhibit the energy gap which can be substantially reduced upon switching (e.g. by external magnetic field) to ferromagnetic configuration. Based on this we predict that the structure will exhibit magnetoresistive effect, which makes graphene nanomeshes of this kind relevant for spintronic applications.
\end{abstract}

DOI: 10.12693/APhysPolA.131.830

PACS/topics: 73.22.Pr, 75.75.-c, 72.25.Dc

\section{Introduction}

The enormous interest in graphene, a two-dimensional (2D) honeycomb lattice of carbon atoms discovered in 2004 [1], has both fundamental and practical reasons. The electrons in graphene can be described by effective Hamiltonian formally identical to that of relativistic massless particles (the Dirac fermions) [2]. One consequence of this is that they possess exceptionally high mobilities of $10^{4} \mathrm{~cm}^{2} /(\mathrm{V} \mathrm{s})$ and more $[3,4]$.

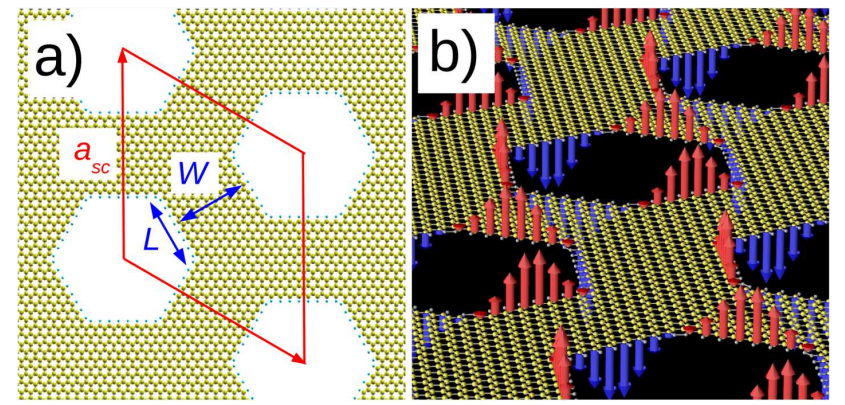

Fig. 1. (a) Example of the structure used in calculations. The marked structural parameters are discussed in the text. (b) The magnetic configuration of the structure in the antiferromagnetic ground state. The red and blue arrows indicate the magnetic moments on the outermost atoms in the lowest energy configuration.

While the fast graphene-based transistors have already been demonstrated [5-8], such devices typically suffer from poor separation of their on and off states. The

\footnotetext{
* corresponding author; e-mail: maciej.zwierzycki@ifmpan.poznan.pl
}

$I_{o n} / I_{o f f}$ ratio of the collector currents is at most of the order of 10 in graphene devices [8] (compared to $10^{4}$ and more in Si MOSFETs), which seriously hinders the prospect of their application in logical circuits. This poor performance is caused by the absence of the gap in graphene electronic structure.

One way of introducing the gap into graphene is to pattern it into ribbons. The combination of confinement and interaction effects leads then to the opening of the gap which scales as $\Delta E \sim 1 / W$ with the width $(W)$ of the ribbon $[9,10]$. In order to generate the band gaps in the range of $0.1 \mathrm{eV}$, necessary for practical applications, the width in the sub $10 \mathrm{~nm}$ range is needed. Such widths are accessible via various manufacturing processes, however, two problems arise. Firstly, the scattering at imperfect edges can lead to significant reduction of carrier mobility thus rendering the device non-competitive with the current Si based transistors. Secondly, the maximum current through the narrow nanoribbon is bound to be limited because of the finite number of modes available for conduction. While the first problem can be hopefully overcome thanks to the rapid progress of manufacturing techniques [11], the second is of more fundamental nature.

Another kind of nanostructure exhibiting a nonzero gap is graphene nanomesh, i.e. graphene patterned with a high density array of antidots (holes). When the stripes of graphene in between neighboring antidots ("necks") are sufficiently narrow the band gap opens, just like in the case of single nanoribbon. Nanomesh based transistors have been shown to exhibit improved $I_{\text {on }} / I_{\text {off }}$ ratio while being able to carry currents up to 100 times larger than their nanoribbon based counterparts [12, 13].

Of various possible antidot shapes, hexagonal one is particularly interesting as it features, when properly oriented with respect to the underlaying graphene lattice, internal zigzag edges. In nanoribbons such edges 
can exhibit magnetic moments $[14,15]$ with the exchange interaction between moments at opposite edges favoring antiferromagnetic (AF) configuration [16]. The persistence of the edge magnetism up to room temperatures in carefully prepared samples was demonstrated experimentally in [17]. The electronic structure of the ground state features the band gap, which closes when the magnetic configuration is changed to ferromagnetic $(\mathrm{F})$ one. The aim of the present paper is to study electronic and magnetic properties of graphene nanomesh with hexagonal antidots. The specific purpose is to see if the ground state supports spin polarization with the configuration amenable to manipulation by external magnetic field. The existence of such, antiferromagnetic, configuration would indicate the possibility of measuring magnetoresistive effect.

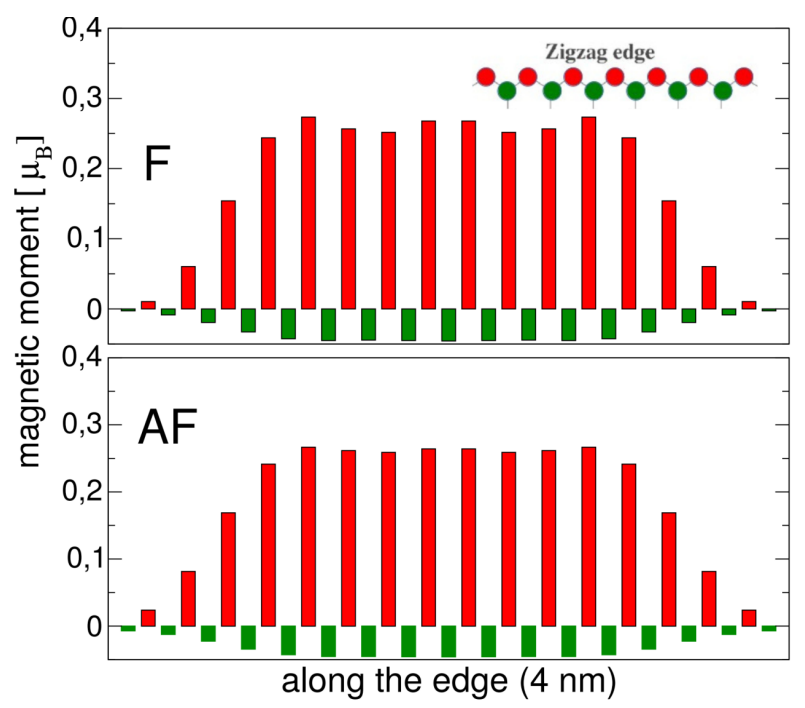

Fig. 2. The magnetic moments along the single inner edge in ferromagetic (top) and antiferromagnetic (bottom) configuration. The red bars correspond to the outermost atoms of the zigzag edge, as marked in the sketch in the top part.

\section{The method}

The calculations were carried out for graphene nanomesh shown in Fig. 1a, using density functional tight binding (DFTB) method [18] as implemented in DFTB+ code [19]. The orientation of the antidots was always chosen so as to produce perfect internal zigzag edges. The dangling edge bonds were saturated with hydrogen atoms. The geometry of the system can be characterized by the following parameters (see Fig. 1a): $a_{s c}$ the supercell lattice constant (equivalent to the distance between the centers of the neighboring antidots), $L$ the length of the inner zigzag edge and $W$ - the width of the neck between neighboring antidots. The positions of carbon atoms within the supercell were initially chosen according to the bulk graphene lattice constant $\left(a_{g r}=1.427 \sqrt{3} \AA\right.$ as determined in separate calculation). The edge lengths $(L)$ ranged between 20 and $60 \AA$, which corresponds to between 8 and 24 outermost carbon atoms. The supercell lattice constants $\left(a_{s c}\right)$ were between 55 and $122 \AA$. Once the unit cell was set up, its global parameters were not changed anymore. The internal geometry however was optimized during the self-consistent calculations. For performance sake (the unit cells contained $10^{3}$ and more atoms) the relaxation was restricted to the hydrogens and the two lines (zigzags) of carbon atoms next to the edge. The self-consistent calculations were performed using single point in the supercell Brillouin zone (BZ), which corresponds to several hundred or more points for the original $1 \times 1$ cell.

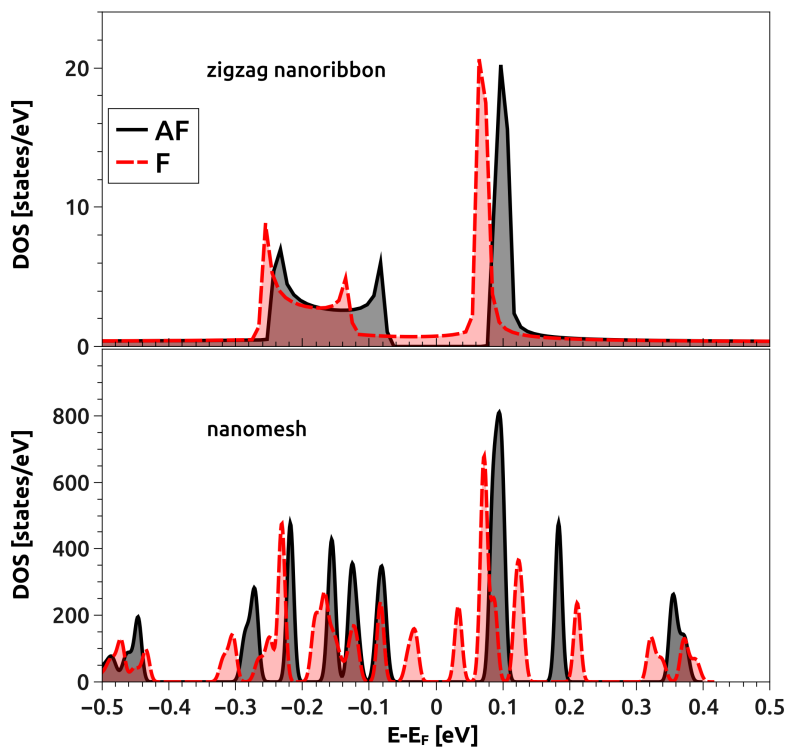

Fig. 3. Density of states (DOS) of zigzag edged nanoribbon (top) and of the nanomesh (bottom). Results for antiferromagnetic and ferromagnetic state are marked with black (solid line) and red (dashed line) color respectively.

\section{The results}

For all the structures considered it has been found that the ground state with antiferromagnetic (AF) ordering of magnetic moments at different edges was energetically favorable, compared to para- and ferromagnetic (F) solutions. The example of magnetic structure is shown in Fig. 1b. Note that the antiferromagnetic alignment of magnetic moments holds both between edges within the same antidot and across the neck dividing the antidots. The magnetic moments are located mostly at the outermost carbon atoms. The maximum values of the moments, at the center of the edge, depend weakly on the length of the edge but falls generally around $0.25 \mu_{\mathrm{B}}$ per atom, that is close to the value predicted for infinite zizgag nanoribbons. The magnetic moments for the atoms 
along the single edge in both ferro- and antiferromagnetic configuration are presented in Fig. 2 for nanomesh with $a_{s c}=88 \AA$ and the length of the inner edge equal to $L=40 \AA$ (corresponding to 16 outermost atoms) and the neck width $W \approx 20 \AA$. The same structural parameters were used for Fig. 3. The red bars correspond to the outermost atoms (see the inset).

With the nature of the ground state established, we would now like to see whether its electronic structure exhibit a gap by studying the density of states (DOS). The DOS for the nanomesh is shown in bottom part of Fig. 3. In the top panel we show, for comparison, the DOS of zigzag nanoribbon with the width equal to the width of the nanomesh's neck. The nanoribbon's DOS for AF configuration exhibits a well defined band gap of about $0.16 \mathrm{eV}$ which closes in $\mathrm{F}$ configuration. The nanomesh DOS exhibits additional modulation because of the confinement effects introduced by antidots. However, the same basic features can be observed for both structures. In particular, there exists a similarly sized gap in AF configuration. In $\mathrm{F}$ configuration there exists, for nanomesh, a small band gap right at the Fermi level. There are, however, states leading to the finite DOS already 20-30 meV away form the Fermi level which in present calculations corresponds to neutral samples. While the details of electronic structure depends on the geometry of the nanomesh, i.e. the combination of $a_{s c}, L$ and $W$, the overall picture outlined above remains unchanged. There is always a gap in the AF configuration and switching to ferromagnetic state introduces states into the vicinity of the Fermi level. As the shifts of the Fermi energy in the range of tens of meV correspond to carrier densities easily achievable in experiment (e.g. by gating) it enables one to envisage the situation when the system can be switched between insulating and conducting states by manipulating its magnetic configuration.

\section{Conclusion}

We have studied electronic and magnetic structure of graphene nanomeshes with hexagonally shaped antidots. We have found that the ground state of the system is antiferromagnetic and its electronic structure features the band gap. The gap is substantially reduced upon switching (e.g. by external magnetic field) to ferromagnetic configuration. The precise amount of the reduction depends strongly on the geometry (the values of $a_{s c}, L$ and $W$ ) of the system but invariably one finds regions of finite DOS within the former gap, usually in the vicinity of the Fermi level. This change in electronic structure should be reflected in the transport properties, manifesting as magnetoresistive effect.

\section{Acknowledgments}

This project was supported by the Polish National Science Centre from funds awarded through the decision No. DEC-2013/10/M/ST3/00488.

\section{References}

[1] K.S. Novoselov, A.K. Geim, S.V. Morozov, D. Jiang, Y. Zhang, S.V. Dubonos, I.V. Grigorieva, A.A. Firsov, Science 306, 666 (2004)

[2] A.H. Castro Neto, F. Guinea, N.M.R. Peres, K.S. Novoselov, A.K. Geim, Rev. Mod. Phys. 81, 109 (2009).

[3] K.I. Bolotin, K.J. Sikes, J. Hone, H.L. Stormer, P. Kim, Phys. Rev. Lett. 101, 096802 (2008).

[4] X. Du, I. Skachko, A. Barker, E.Y. Andrei, Nat. Nanotechnol. 3, 491 (2008).

[5] Y.-M. Lin, K.A. Jenkins, A. Valdes-Garcia, J.P. Small, D.B. Farmer, P. Avouris, Nano Lett. 9 , $422(2009)$.

[6] L. Liao, Y.-C. Lin, M. Bao, R. Cheng, J. Bai, Y. Liu, Y. Qu, K.L. Wang, Y. Huang, X. Duan, Nature 467, 305 (2010).

[7] Y.-M. Lin, A. Valdes-Garcia, S.-J. Han, D.B. Farmer, I. Meric, Y. Sun, Y. Wu, C. Dimitrakopoulos, A. Grill, P. Avouris, K.A. Jenkins, Science 332, 1294 (2011).

[8] F. Schwierz, Nat. Nanotechnol. 5, 487 (2010).

[9] Y.-W. Son, M.L. Cohen, S.G. Louie, Phys. Rev. Lett. 97, 216803 (2006).

[10] M.Y. Han, B. Özyilmaz, Y. Zhang, P. Kim, Phys. Rev. Lett. 98, 206805 (2007).

[11] J. Bai, Y. Huang, Mater. Sci. Eng. R Rep. 70, 341 (2010).

[12] J. Bai, X. Zhong, S. Jiang, Y. Huang, X. Duan, Nat. Nanotechnol. 5, 190 (2010).

[13] J. Yang, M. Ma, L. Li, Y. Zhang, W. Huang, X. Dong, Nanoscale 6, 13301 (2014).

[14] C. Tao, L. Jiao, O.V. Yazyev, Y.-C. Chen, J. Feng, X. Zhang, R.B. Capaz, J.M. Tour, A. Zettl, S.G. Louie, H. Dai, M.F. Crommie, Nat. Phys. 7, 616 (2011).

[15] K. Tadyszak, M. Maćkowiak, M.A. AugustyniakJabłokow, R. Strzelczyk, J. Mol. Struct. 1076, 31 (2014).

[16] L. Pisani, J.A. Chan, B. Montanari, N.M. Harrison, Phys. Rev. B 75, 064418 (2007).

[17] G.Z. Magda, X. Jin, I. Hagymasi, P. Vancso, Z. Osvath, P. Nemes-Incze, C. Hwang, L.P. Biro, L. Tapaszto, Nature 514, 608 (2014).

[18] M. Elstner, D. Porezag, G. Jungnickel, J. Elsner, M. Haugk, T. Frauenheim, S. Suhai, G. Seifert, Phys. Rev. B 58, 7260 (1998).

[19] B. Aradi, B. Hourahine, T. Frauenheim, J. Phys. Chem. A 111, 5678 (2007). 